\title{
Delayed surgery of locally advanced low rectal cancer after preoperative concurrent chemo-radiotherapy, trial for sphincter- saving radical resection with good local control
}

\author{
Osama Mahmoud, ${ }^{a} M D$, FRCS; Hesham Maged, ${ }^{a} M D$; Gamal Fawzy, ${ }^{a} M D$; \\ Ahmed Hussein, ${ }^{a} M D$; Ramy Ghali, ${ }^{b} M D$; Khaled El-Ghoneimy, ${ }^{b} M D$
}

a) Department of General Surgery, Ain Shams University, Cairo, Egypt.

b) Department of Clinical Oncology, Ain Shams University, Cairo, Egypt.

\begin{abstract}
To evaluate multimodality treatment therapy in patients with locally advanced low rectal adenocarcinoma as a trial for sphincter-saving radical resection with good local control. Twenty two patients underwent preoperative concurrent chemoradiotherapy, (CCRT) and radical resection for locally advanced low rectal adenocarcinoma. Patients received preoperative bolus 5-fluorouracil (5-FU) and leucovorin concurrent with radiotherapy to a total dose of $50.4 \mathrm{~Gy}$, followed by radical resection surgery 6 weeks after CCRT. Fifty-five percent of patients achieved tumor downstaging, $14 \%$ of patients showed pathological complete remission, sphincter-saving rate was $82 \%$, and there were no deaths related to preoperative CCRT or surgery. Mild hematological and gastrointestinal toxicity was noted during the course of preoperative CCRT. Overall, 3-year survival rate was 78\% and the rate of locoregional recurrence was $13.6 \%$. This study showed that many patients with locally advanced rectal cancer can be operated on with sphincter-saving radical resection surgery under good local control after preoperative CCRT which induces tumor downstaging.
\end{abstract}

\section{Introduction:}

Carcinoma of the colon and the rectum is considered the third most common malignant neoplasm in Egypt and shares the epidemiological characteristics of developing countries which are higher incidence in the younger with an estimated annual incidence of more than 4000 new cases. ${ }^{1}$ Carcinoma of the rectum predominates, of which $70 \%$ are limited to the sigmoid colon and rectum. ${ }^{2}$ Rectal cancer accounts for an annual mortality of about 77,000 patients worldwide. ${ }^{1}$ Radical resection of the primary lesion is the treatment of choice for rectal cancer, but for locally advanced low rectal cancer $(0-6 \mathrm{~cm}$ from anal verge), management is difficult either because of the possibility of inadequate removal of safety margins with great incidence of local recurrence or inability to preserve the anal sphincter. ${ }^{3}$ Neoadjuvant therapy usually induces tumor downstaging, preoperative application of radiotherapy alone or combined with chemotherapy (radio-sensitizing effect of the chemotherapy) for a low rectal tumor can induce tumor regression, and further surgery can be performed with feasibility of complete resection with adequate safety margins. ${ }^{4-7}$ This regimen significantly increases the rate of sphinctersaving operations, enhances local control and/or overall survival is prolonged. ${ }^{8-10}$ The tumor stage in Dukes' classification or (TNM) system is the most important prognostic factor to determine the response of locally advanced low rectal cancer to preoperative CCRT. 9,11

\section{Patients and methods:}

This study involved 22 patients with locally advanced low rectal carcinoma treated with preoperative CCRT followed by radical resection in the Departments of General Surgery and Clinical Oncology, Ain Shams University, in the period from October 2006 to April 2008. Locally advanced cancer was defined as T3 (tumor invades through muscularis propria into 
subserosa to peri-colic or peri-rectal tissues), while T4 (tumor perforates the visceral peritoneum or directly invades other organs or structures). Low rectal cancer as defined as tumors located $(0-6 \mathrm{~cm})$ from the anal verge. All patients were clinically staged to be locally advanced low rectal cancer by physical examination, proctoscopy, flexible colonoscopy, endo-rectal ultrasound, computed tomography (CT), and/or magnetic resonance imaging (MRI). Confirmation of the pathology was performed by biopsy and histopathological examination of rectal lesion in all patients. Exclusion of distant metastases was done by chest X-ray, ultrasound and CT. Moreover, complete blood count (CBC) and carcinoembryonic antigen (CEA) were performed before treatment.

\section{Preoperative radiotherapy:}

Radiation was delivered with 6 and/or 15MV photon beams, while the patient was in the prone position using a three-fields radiation technique (Post pelvic field, Rt lateral and Lt lateral pelvic fields). The treatment dose was 50.4 Gy in 28 fractions for 5 weeks. All fields were treated daily. The superior border was at the L5-S1 interspace, and the inferior border was $3 \mathrm{~cm}$ below the primary tumor or at the inferior aspect of the obturator foramen, whichever is the most inferior. The Lateral border extended $1.5 \mathrm{~cm}$ beyond the true bony pelvis. The Lateral fields were $1-1.5 \mathrm{~cm}$ behind the anterior bony sacral margin posteriorly and at the posterior margin of the symphysis pubis anteriorly in T3 disease (to treat only the internal iliac nodes) or at the anterior margin of the symphysis pubis in T4 disease(to treat the external iliac nodes). Blocks were used to spare the posterior muscles and soft tissues behind the sacrum, to reduce the amount of dose inferior to the symphysis pubis and to decrease the amount of small bowel both superiorly and inferiorly.

\section{Preoperative chemotherapy:}

Concurrent chemotherapy with fluorouracil $400 \mathrm{mg} / \mathrm{m} 2 / \mathrm{d}$, together with leucovorin 20 $\mathrm{mg} / \mathrm{m} 2 / \mathrm{d}$ during 4 days, was administered during the first and fifth week of radiotherapy. All patients should receive adjuvant chemotherapy with the same fluorouracil/leucovorin regimen.

\section{Surgery:}

The median interval between completion of radiotherapy and surgery was 6 weeks (range, 5 - 9 weeks). Pre-operative colon preparation was performed to all patients using both chemical 3 days before and mechanical methods 1 day before the radical resection. Surgery was performed through conventional open method starting by transverse lower abdominal incision or midline abdominal incision, mobilization of the left colon, sigmoid colon, rectum with total mesorectal excision, up to anal canal. Curative resection was defined as a one-stage procedure of complete removal of tumors followed by end to end anastomosis using curved endo-anal stapler 29-33 F, or abdomino-perineal resection (APR). Excision of the pathological segment of the rectum with free safety margins, marked $3-5 \mathrm{~cm}$ from the lesion, the resected margins were examined by frozen section to ensure free anastomotic edges from any remnant malignant tissues. All resected specimens were fixed immediately after surgery in formalin, and examined by pathologists in the same hospital.

\section{Follow-up \& statistical analysis:}

The median follow-up was 12.5 months (range, 5.0-37.0 months), with intervals of 3 to 6 months. Clinical physical examination and laboratory surveys were performed to all patients including CEA, chest X-ray, and liver ultrasound, which were followed up every 3 months. Flexible colonoscopy was performed every 6 months. Loco-regional recurrence was also defined as a recurrence occurring at the intra-abdominal or intrapelvic cavity, anastomotic segment, tumor bed, peritoneum, and regional lymph nodes. Survival rate was estimated by the Kaplan-Meier method.

\section{Results:}

The study involved 22 patients with locally advanced low rectal adenocarcinoma. The median age of patients was 61 years (range, $28-76$ years), 16 females $(72.7 \%$ ) and 6 males $(27.3 \%)$. Staging was done before and after CCRT by TNM classification system of the 
American Joint Committee of Cancer, Table(1). 6 patients staged as T3N0 (27.3\%), 1 patient T4N0 (4.5\%), 10 patients T3N1 (45.5\%), 1 patient T4N1 $(4.5 \%)$, and 4 patients $\mathrm{T} 3 \mathrm{~N} 2$ (18.2\%) Table(1). After completing the courses of preoperative CCRT, the tumor stage was
TONO in three patients (13.6\%), T2N0 in five patients (22.7\%), T3N0 in six patients (27.3\%), T4N0 in one patient $(4.5 \%)$, T0N1 in one patient $(4.5 \%), \mathrm{T} 1 \mathrm{~N} 1$ in one patient, $\mathrm{T} 3 \mathrm{~N} 1 \mathrm{in}$ one patient $(4.5 \%)$, T0N2 in one patient $(4.5 \%)$, and T3N2 in three patients (13.6\%) Figure(1).

Table (1): Shows TNM before and after CCRT.

\begin{tabular}{|c|c|c|c|c|}
\hline \multirow{2}{*}{ TNM } & \multicolumn{2}{|c|}{ Before CCRT } & \multicolumn{2}{c|}{ After CCRT } \\
\cline { 2 - 5 } & No. & $\%$ & No. & $\%$ \\
\hline T0 N0 & ---- & ---- & 3 & 13.6 \\
\hline T2 N0 & ---- & ---- & 5 & 22.7 \\
\hline T3 N0 & 6 & 27.3 & 6 & 27.3 \\
\hline T4 NO & 1 & 4.5 & 1 & 4.5 \\
\hline T0 N1 & ---- & --- & 1 & 4.5 \\
\hline T1 N1 & ---- & ---- & 1 & 4.5 \\
\hline T3 N1 & 10 & 45.5 & 1 & 4.5 \\
\hline T4 N1 & 1 & 4.5 & ---- & ----- \\
\hline T0 N2 & ---- & --- & 1 & 4.5 \\
\hline T3 N2 & 4 & 18.2 & 3 & 13.6 \\
\hline
\end{tabular}

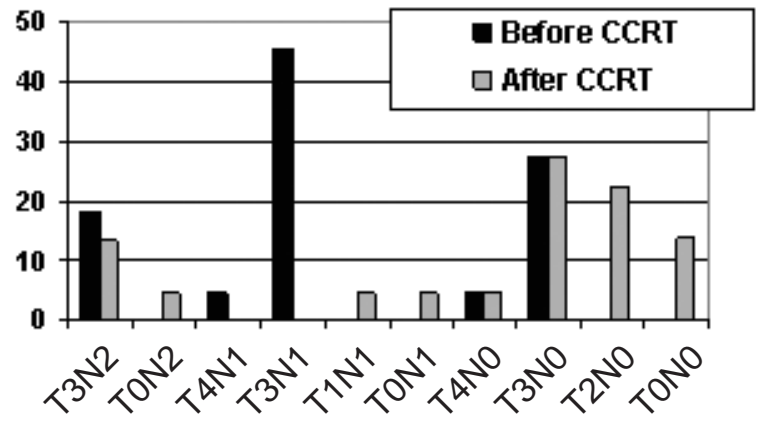

Figure (1)

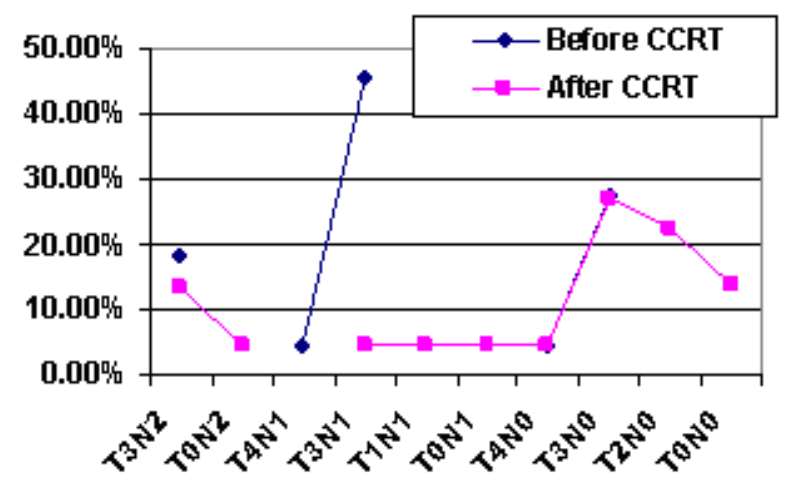

Figure (2) 
After preoperative CCRT, three of six patients in the initial T3N0 stage group, showed downstaging. Also 6 of 10 patients showed downstaging from the initial T3N1 stage group. 2 of 4 patients in the initial T3N2 stage group, and 1 patient in the initial T4N1 stage group. While no change was noted in the initial T4N0 stage group. The downstaging rates were $55 \%$ (12 of 22 patients) Figure(2). Three patients (13.6\%) showed pathological complete regression (T0N0), two from the initial T3N1 stage group and one from the initial T3N2 stage group. Five patients $(22.7 \%)$ noted tumors confined to the bowel wall (T2N0), three from the initial T3N0 stage group, one from the initial T4N1 stage group, and one from the initial T3N2 stage group. Seven patients (31.8\%) had extra-rectal extension of disease without nodal involvement (T3N0\&T4N0), two from the initial T3N0 stage group, one from the initial T4N0 stage group, and four from the initial T3N1 stage group. The remainder noted as tumors with positive nodal metastasis, Table(1).

\section{Outcome:}

All 22 patients underwent potentially curative surgical resection, with total mesorectal excision. 18 patients $(81.8 \%)$ operated by low anterior resection surgery (LAR), and 4 patients (18.2\%) operated by abdomino-perineal resection surgery (APR) Figure(3). Sphinctersaving procedure was $82 \%$ (18 patients). Early complications were noted in two patients (9\%), both were classified as wound infection. Later complications required an additional surgical procedure in two patients (9\%), two patients noted leakage of anastomosis, one on the 5th day and the other on the 8th day postoperatively. The cause of the leakage was the ischemia of the distal stump due to excessive dissection distally, both were explored and underwent permanent diverting colostomy.

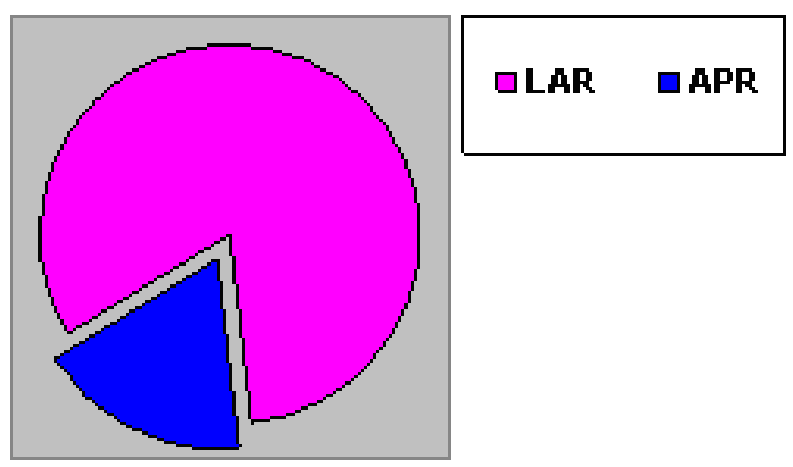

Figure (3)

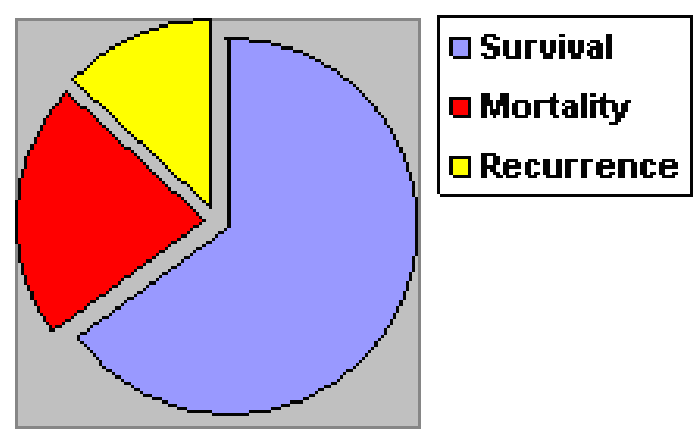

Figure (4) 
The patients were seen at least weekly during the multimodality regimen. No mortality related to either CCRT or to the surgical procedure was observed. The overall 3-year survival rate was up to $78 \%$. Five patients died all over the course of follow up, three from disseminated malignancy related mortality, one from unexplained cause of death, and the 5th lost during the follow up and considered died. After curative resection, three patients (13.6\%) showed loco-regional recurrence Figure(4). Most of patients had either grade 1 or 2 hematological or gastrointestinal toxicity, only two patients experienced grade 3 diarrhea and hospitalization was not required for such patients with acute toxicity and improved by home therapy.

\section{Discussion:}

The management of rectal cancer showed significant revolution over the past decade with improvement in both surgical technique and adjuvant therapies. ${ }^{12}$ The progression of surgical management has been of particular interest, as surgery is the only potentially curative treatment. The major goals of surgery are to optimize oncologic outcome and maintain anorectal and genitourinary function. ${ }^{10,13} \mathrm{In}$ the last few years there is much progress in medical oncology and developing of new chemo-therapeutic drugs with more efficacy and less side effects, also showed up new machines with safer technique of radiotherapy. $7,14,15$ All of the above made the local control of advanced low rectal cancer feasible and more effective than before. In this study, we tried to get the maximum benefits of the efficacy of preoperative CCRT combined with radical resection surgery in patients with locally advanced low rectal carcinoma hoping to get better results. The results were satisfactory as regard CCRT before surgery as it could induce tumor downstaging, improve sphincter-saving rates, with good local control of the disease.

Preoperative radiotherapy combined with total mesorectal excision (TME) has provided excellent local control in the treatment of rectal cancer. Lateral pelvic and perirectal lymph node metastases were independent risk factors for local recurrence. ${ }^{9,10}$ The proportional hazard model showed that age, grade, depth of the tumor invasion, perirectal, and lateral pelvic lymph node metastasis to be independent prognostic factors. Safety margins are the most important prognostic factor after surgical resection for locally advanced rectal carcinoma. Preoperative CCRT induces tumor regression with reduction of its size, induces downstaging, and gives a chance for complete resection of the rectal cancer $7,19,20$. This study showed a significant $55 \%$ of both tumor and nodal downstaging and $68 \%$ of either tumor or nodal downstaging. Tumor regression result is comparable with the reported benefit of preoperative radiotherapy only (18-26\%), and reported benefit of preoperative chemoradiotherapy (53-76\%).

Despite recent advances in radiation and chemotherapy, surgical resection remains an integral part of curative therapy for this disease. The anatomic complexity of the pelvis makes therapy for this disease considerably more complicated. Local recurrence is also a greater concern in rectal cancer than in colon cancer. ${ }^{3,11}$ The choice of surgical therapy depends on the location of the tumor, depth of rectal wall invasion, and clinical stage. ${ }^{17,18,21}$ In this study, 18 patients $(81.8 \%)$ were operated on by low anterior resection (LAR), and 4 patients, $(18.2 \%)$ were operated on by abdominoperineal resection (APR). Sphincter-saving procedure was $82 \%$ (18 patients) Figure(3).

The results were compared with similar studies carried out in different centers to determine any changes in treatment methods, recurrence rates, and survival. The local recurrence rate was $13.6 \%$ compared with $12.7 \%$ in a study done in Roswell Park Cancer Institute. ${ }^{22}$ Stockholm and Swedish Rectal Cancer trials have found that, compared with surgery alone, preoperative radiation not only decreases local recurrence (11\% versus $27 \%$ ) but also increases overall survival ( $58 \%$ versus 48\%). ${ }^{23,24}$ Many studies showed that patients treated with preoperative radiotherapy had a low recurrence rate, on the other hand the type of surgical therapy had no significant effect on local recurrence. $2,25,26$ There was no significant change in overall survival between the present study and other studies. Although some authors had different results in overall 
survival, our series showed a local recurrence rate of $13.6 \%$ and overall 3-year survival rate up to $78 \%$.

Sphincter-saving procedures performed for low rectal cancer had local recurrence rates of 4-17\%. Ruller et al. published excellent results of a study in which all tumors were $\mathrm{T} 3$ lesions, and after using preoperative chemoradiotherapy, the rate of sphincter-saving resection was $78 \%$ for lesions located 2-6 cm from the anal verge initially, and the local recurrence rate was $2 \% .15$ Indeed, the results of our series showed that the rate of sphincter saving was $82 \%$, and the local control rate was more than $86 \%$, using multimodality regimens for locally advanced low rectal carcinoma.

Using preoperative irradiation alone followed by radical surgery 6-8 weeks later has great influence on downstaging which was improved, ${ }^{10,20}$ while adjuvant chemotherapy alone showed improvement in survival rate.5,6 So combined chemotherapy with preoperative radiation may improve both local control and dissemination of the disease. ${ }^{5,11}$ In this study the local control was up to $86 \%$ and overall survival rate was $78 \%$ using similar multimodality regimens for locally advanced rectal disease. The most common grade 3 toxicity induced by preoperative CCRT encountered in our study was diarrhea which was recorded in $9 \%$ of patients, all of them continued treatment and no grade 4 toxicity was noted. These results are similar to published reports that used similar regimens of CCRT.

\section{Conclusion:}

Patients with locally advanced low rectal carcinoma may benefit from preoperative CCRT. The obtained results showed that, the multi-modality treatment has induced significant tumor downstaging, increased radical resection with free margins, encouraged sphincter-saving procedure, and improved the local control rate with a low rate of complications. Although chemotherapy and radiation are now frequently recommended in conjunction with surgical therapy, further randomized controlled trials for preoperative CCRT should be performed to establish the guidelines for these protocols.

\section{References:}

1- El-Attar I: Colorectal cancer: Magnitude of the problem. National Cancer Institute, Annual Cancer Conference of Egyptian Cancer Society, Cairo University, Cairo, Egypt 2005.

2- Medich D, McGinty J, Parda D, Karlovits S, Davis C, Caushaj P, et al: Preoperative chemoradiotherapy and radical surgery for locally advanced distal rectal adenocarcinoma: Pathologic findings and clinical implications. Dis Colon Rectum 2001; 44: 1123-1128.

3- Leo E, Belli F, Andreola S, Gallino G, Bonfanti G, Ferro F, et al: Total resection and complete mesorectum excision followed by coloendoanal anastomosis as the optimal treatment for low rectal cancer: The experience of the National Cancer Institute of Milano. Ann Surg Oncol 2000; 7: 125-132.

4- Glimelius B, Isacsson U, Jung B, Pahlman L: Radiotherapy in addition to radical surgery in rectal cancer: Evidence for a dose-response effect favoring preoperative treatment. Int J Radial Oncol Biol Phys 2007; 37: 281-287.

5- Gunderson LL: Indication for and results of combined modality treatment of colorectal cancer. Acta Oncol 1999; 38: 721.

6- Krook JE, Moertel CG, Gunderson LL, Wieand HS, Collins RT, Heart RW, et al: Effective surgical adjuvant therapy for highrisk rectal carcinoma. $N$ Eng $J$ Med 1991; 324: 709-715.

7- Vini L: Neoadjuvant radiochemotherapy for rectal cancer. Dig Dis 2007; 25: 56-66.

8- Gérard JP, Conroy T, Bonnetain F, Bouché $\mathrm{O}$, Chapet $\mathrm{O}$ et al: Preoperative radiotherapy with or without concurrent fluorouracil and leucovorin in T3-T4 rectal cancers: Results of FFCD 9203. Journal of Clinical Oncology 2006; 24: 4620-4625.

9- Kaminsky-Forrett MC, Conroy T, Luporsi E, Peiffert D, Lap-eyre M, Boissel P, et al: Prognostic implication of downstaging following preopeartive radiation therapy for operable T3-T4 rectal cancer. Int $f$ Radiat Oncol Biol Phys 1998; 42: 935-941. 
10-Marks G, Mohiuddin M, Masoni L: The reality of radical sphincter preservation surgery for cancer of the distal $3 \mathrm{~cm}$ rectum following high-dose radiation. Int J Radiat Oncol Biol Phys 1993; 27: 779-783.

11-Mohiuddin M, Hayne M, Regine WF, Hanna N, Hagihara PF, McGrath P, et al: Prognostic significance of post chemoradiation stage following preoperative chemotherapy and radiation for advanced/recurrent rectal cancers. Int $J$ Radiat Oncol Biol Phys 2000; 48: 10751080.

12-Chari RS, Tyler DS, Anscher MS: Preoperative radiation and chemotherapy in the treatment of adenocarcinoma of the rectum. Ann Surg 1995; 221: 778-787.

13-McAnena OJ, Heald RJ, LockhartMummery HE: Operative and functional results of total mesorectal excision with ultra-low anterior resection in the management of carcinoma of the lower one-third of the rectum. Surg Gynecol Obstet 1990; 170: 517-521.

14-McArdle CS, Hole D: Impact of variability among surgeons on postoperative morbidity and mortality and ultimate survival. $B M J$ 2006; 302: 1501-1505.

15-Ruller E, Goffre B, Bonnel C, Zerbib F, Caudry M, Saric J: Preoperative radiochemotherapy and sphincter-saving resection for T3 carcinomas of the lower third of the rectum. Ann Surg 2001; 235: 633-640.

16-Gamagami RA, Liagre A, Chiotasso P, Istvan G, Lazorthes F: Colo-anal anastomosis for distal third rectal cancer. Prospective study of oncologic results. Dis Colon Rectum 1999; 42: 1272-1275.

17-Horgan AF, Finlay IG: Preoperative staging of rectal cancer allows selection of patients for preoperative radiotherapy. Br J Surg 2000; 87: 575-579.

18-Kapiteijin E, Marijnen CA, Nagtegaal ID, Putter H, Steup WH, Wiggers T, et al: Preoperative radiotherapy combined with total mesorectal excision for resectable rectal cancer. N Eng Med 2001; 345: 638646.

19-Grann A, Minsky BD, Cohen AM, Saltz L, Guillem JG, Paty PB, et al: Preliminary results of preoperative 5-fluorouracil (5FU), low dose leucovorin, and concurrent radiation therapy for resectable $\mathrm{T} 3$ rectal cancer. Die Colon Rectum 1997; 40: 515522.

20-Mohiuddin M, Regine WF, Marks GJ, Marks JW: High-dose preoperative radiation and the challenge of sphincterpreservation surgery for cancer of the distal $2 \mathrm{~cm}$ of the rectum. Int J Radiat Oncol Biol Phys 1998; 40: 569-574.

21-Rich TA, Skibber JM, Ajani JA, Buchholz DJ, Cleary KR, Dubrow RA, et al: Preoperative infusional chemoradiation therapy for stage T3 rectal cancer. Int $J$ Radial Oncol Biol Phys 1995; 32: 10251029.

22-Roswell Park Cancer Institute: The University at Buffalo, State University of New York, Buffalo, NY 14263, USA, 2005.

23-Stockholm Rectal Cancer Study Group: Randomized study on preoperative radiation in rectal carcinoma. Ann Surg Oncol 1996; 3: 423-430.

24-Swedish Rectal Cancer Trial: Improved survival with preoperative radiotherapy in resectable rectal cancer. $N$ Engl J Med 1997; 336: 980-987.

25-Wagman R, Minsky BD, Cohen AM, Guillem JG, Paty PP: Sphincter preservation in rectal cancer with preoperative radiation therapy and coloanal anastomosis: Long term follow-up. Int Radiat Oncol Biol Phys 1998; 42: 51-57.

26-Wichmann MW, Milller C, Meyer G, Strauss T, Hornung HM, Lau-Werner U, et al: Effect of preoperative radiochemotherapy on lymph node retrieval after resection of rectal cancer. Arch Surg 2002; 137: 206210. 